Article

\title{
Ultrasounds Used as Promoters of Heat-Transfer Enhancement of Natural Convection in Dielectric Fluids for the Thermal Control of Electronic Equipment
}

\author{
Carlo Bartoli ${ }^{1}$, Alessandro Franco ${ }^{1, *}$ (1) and Massimo Macucci ${ }^{2}$ \\ 1 Department of Energy, Systems, Territory and Constructions Engineering (DESTEC), University of Pisa, \\ 56122 Pisa, Italy; carlo.bartoli@unipi.it \\ 2 Department of Information Engineering (DII), University of Pisa, 56122 Pisa, Italy; massimo.macucci@unipi.it \\ * Correspondence: alessandro.franco@ing.unipi.it; Tel.: +39-050-2217154
}

Received: 8 April 2020; Accepted: 10 May 2020; Published: 12 May 2020

check for updates

\begin{abstract}
We present an experimental investigation of the effect of ultrasound application to increase the heat-transfer coefficient for natural convection of a dielectric fluid. An experimental analysis is carried out to estimate the increase of the convective heat-transfer coefficient between an electronic board and a refrigerant fluid, the Fluorinert Electronic Fluid FC-72. For this purpose, an experimental apparatus composed of an electronic board, its electronic control circuit, and data acquisition systems have been designed and implemented. The data collected appear to confirm in some situations of practical interest the enhancement effect of the convective heat-transfer coefficient in connection with the use of ultrasound. The most favorable condition was observed with the fluid in quite low subcooled conditions.
\end{abstract}

Keywords: heat transfer enhancement; acoustic fields; vibrations; thermo-fluid dynamics; experimental analysis; subcooled liquid

\section{Introduction}

The positive influence that ultrasounds have on the enhancement of the convective heat transfer coefficient has been investigated since the 1930s. The first instance in which the topic was taken into consideration is in an old paper by Martinelli and Boelter [1].

However, it is in the 1960s-1970s that significant research activity on the subject began, with experimental and theoretical efforts. In such a period, interesting contributions on the topic were provided by Fand et al. [2], Kubanskii, [3], Holman and Mott-Smith [4], who studied a gas in the presence of natural convection, and Anantanarayanan and Ramachandran [5], Jackson et al. [6], Lemlich and Hwu [7], Fand and Cheng [8], investigated a gas in the presence of forced convection.

In the same period, Larson and London [9], Deaver et al. [10], Fand [11], investigated a liquid in the presence of natural convection, while West and Taylor [12], Scanlan [13], Raben [14] and Bergles [15] focused on liquid in the presence of forced convection; in the same period, Isakoff [16], and Nangia et al. [17], achieved interesting results with a liquid in pool boiling. The use of ultrasonic enhancement in pool-boiling fluids was considered particularly interesting, as demonstrated by further contributions available in papers such as [18-20].

In general, studies show that, in particular conditions, the heat-transfer coefficient can be increased up to 10 times. However, it is also evident from the aforementioned studies that, under suboptimal conditions in terms of ultrasound frequency, or geometric configuration, or state of the fluid, such heat-transfer enhancement may be of the order of the experimental error and, therefore, undetectable. 
In recent years, studies on the enhancement effect of ultrasound were further pursued; they can be divided into two main categories: one focusing on the phenomenology of the interaction of ultrasound with the thermal convective heat transfer coefficient of various fluids, the other focusing on energy efficiency, with the aim of increasing the convective heat transfer coefficient in heat exchangers and of fouling reduction. Various researchers [21,22] have pursued reduction of fouling in order to limit the number of stops needed for cleaning operations.

Thus, it is apparent that a lot of interesting experimental data and associated analysis are available in the literature. A short summary of the existing results is available in recent papers by two of the authors $[23,24]$.

From the available literature, it is apparent that the complexity of the topic makes its treatment at the mathematical and physical levels quite difficult. The complexity of thermos-fluid dynamics is added to that of the acoustic phenomenon and, in addition, there is a non-linear interaction between the two. In order to obtain quantitatively accurate results, one should therefore consider specific models that are as close as possible to the final application.

One of the authors of the present paper has studied ultrasounds used as promoters of convective heat transfer in the perspective of "Enhancement heat transfer" [25]. He investigated the basic physical phenomenon focusing on a stainless steel cylindrical tube with a "sliding" thermocouple insulated from the tube by means of a ceramic electric insulator using distilled water as the coolant fluid. First, he studied the behavior of water in the form of a subcooled liquid and of completely developed pool boiling $[25,26]$, but without meaningful results: the increase in convective heat-transfer coefficient was lower than the experimental error (14\%), in particular, of the order of $7 \%$. Even in conditions of "pool boiling", the improvement is of the order of $10 \%$. The same author then investigated water in "subcooled boiling" conditions; in this case, the improvement of the convective heat-transfer coefficient was significant, reaching $60 \%$ more than using distilled water as a working fluid. In particular, he determined that the convective heat-transfer coefficient depended on the sub-cooling temperature gap $\left(\Delta T_{\text {sat }}\right)$ : in optimal conditions, and the value of $\Delta T_{\text {sat }}$ was of the order of $30 \mathrm{~K}$.

Here we further investigate the heat-transfer enhancement of a liquid in subcooled pool boiling conditions: in this regard, the investigation performed by Parker and Bergles [18] can be considered a quite important basic reference. They studied the interaction of ultrasounds at $55 \mathrm{kHz}$ with convection from cylinders in the R-113 fluid in the subcooling and pool boiling conditions.

Here we specifically consider the application of ultrasound to the cooling of electronic components. Heat removal has become one of the most relevant bottlenecks to the further increase of integrated circuit performance. Since around 2004 there has been no significant increase of the clock frequency in microprocessors because the maximum admissible power dissipation per unit surface has been reached, forcing manufacturers to follow different paths to performance increase, such as the fabrication of multiple cores on a single die. This, however, is not as good as raising the clock frequency, and thus the pace at which the computing power increases with time has been reduced, leading to a slowdown of the computer market. Also a very promising technological approach, 3-D integration, which should exploit the third spatial dimension, has seen very limited application, just for devices with low power dissipation, such as relatively slow memories. If devices have to be immersed in the cooling fluid, this has to be an insulating dielectric fluid, in order to avoid leakage currents and to prevent the rise in stray capacitances that would result from an elevated relative permittivity. There are several fluids suitable for such an application. This paper refers to this type of experience aimed to test the increase of the heat-transfer coefficient with ultrasound in one of the specific conditions of interest for the application: liquid fluid in subcooling conditions. In particular, an ultrasonic generator with a $500 \mathrm{~W}$ output power and a constant ultrasound frequency of $40 \mathrm{kHz}$ is used and a specific fluid is adopted as a coolant, the FC72.

\section{The Use of Ultrasound for Heat Transfer Enhancement: Fundamental Physical Elements}

Sound can be considered as a mechanical perturbation from a state of equilibrium that propagates through an elastic material medium such as a gas, a liquid or a solid. Such a perturbation consists of a 
pressure fluctuation characterized by its frequency. If the frequency is less than $20 \mathrm{~Hz}$, we speak of infrasound, if the frequency is between 20 and $20 \mathrm{kHz}$ we have sounds perceptible by the human ear, and finally if the frequency is greater than $20 \mathrm{kHz}$ we are in the field of ultrasound.

Ultrasound is used in various fields. The most relevant is probably the medical sector, in which they are used for a lot of diagnostic examination or for precision and non-invasive surgical operations. But the use of ultrasound is considered in underwater target detection with the use of SONAR, in the non-destructive testing mechanical industry. Considering the chemical sector, an entire strand of research called sonochemistry is actively aimed at studies of the increase in the speed of reactions. Another relevant field of application is probably the biological one, where by means of ultrasound it is possible to effectively extract enzymes and proteins through the lysis of cell membranes of bacteria or cell cultures. Other uses include extracting substances from the soil for analysis and cleaning large-scale objects.

Considering the use for heat transfer, analyzing the various studies in the literature, one can conclude that, in particular in the case of liquids, both in single phase and in two-phase conditions, an ultrasonic field often results in the overall enhancement of the heat-transfer coefficient.

The observed enhancement depends on the characteristics of the fluid, and on different process variables, such as ultrasonic intensity, ultrasonic frequency, temperature and pressure. In Refs. [23,24] the authors have reviewed both the effects of ultrasound in some special applications such as heat exchangers, and in general. In particular, it has been observed that the heat transfer enhancement can be induced by three main different mechanism, determined by acoustic fields, which occur or not depending on the fluid under examination. In some particular cases, often involving liquid, the heat-transfer enhancement can be relevant because all the involved mechanisms interact:

- Acoustic streaming: consists in the alteration of the trajectories of the fluid flow, with its consequent mixing which alters heat transfer;

- Acoustic alteration of the boundary layer: the oscillation of the velocity component perpendicular to the portion of the wall affected by the exchange determines alternating compression and expansion phases of the thermal boundary layer which determines an overall increase of the heat transfer;

- Acoustic cavitation: the phenomenon causes temperature and pressure oscillations due to the propagation of the acoustic waves, which can be quite large, the fluid locally reaches, during the rarefaction phase, the steam condition. Steam bubbles then form: after a certain period of time, they implode violently, causing shock waves and jets of fluid. If the collapse of the bubbles occurs near the thermal boundary layer, it produces strong internal mixing, with consequent variation of the local heat-transfer coefficient.

\subsection{Sound and Acoustic Cavitation}

In the case of ultrasound action in single or two phases fluid, in the presence of dissolved gas, two phenomena alter the heat-transfer coefficient: acoustic streaming in liquid or gas and acoustic cavitation. Cavitation is the most relevant phenomenon. It can be explained as follows: an intense ultrasonic field resulting in a rapid succession of depression and overpressure phases. During the depression phase, it creates bubbles of gas inside the liquid that grow until the phase of acoustic depression (negative pressure) lasts. This formation of microscopic gas bubbles is the beginning of cavitation (i.e., the formation of gaseous cavities inside the liquid). During the following phase of compression, the high pressure exerted on the newly expanded bubble compresses the same by greatly increasing the temperature of the gas contained there as long as the bubble collapses on itself, imploding, resulting in huge release of shock energy. The shock energy caused by the implosion of the gas bubble hits the surface of the object to be cleaned and interacts both physically and chemically.

\subsection{The Mechanisms of Action of Ultrasonic Waves for Heat-Transfer Enhancement}

In general, it can be observed that the action of ultrasound can be one of the possible ways to enhance the heat transfer performances of a liquid flow in the laminar regime up to the level usually 
observed for turbulent flow conditions. A theoretical analysis of the problem and the definition of the heat-enhancement effect of ultrasounds is quite a complex task.

Indeed, the acoustic field propagation can be sensitive to fluid property variations, as well as to the different geometries involved. The heat-transfer enhancement depends in different ways on the following variable and parameters:

- $\quad$ the fluid and its phase (gas, liquid or two-phase);

- $\quad$ the operating conditions of the fluid (such as temperature and pressure);

- the ultrasonic frequency of the generator, $f$;

- $\quad$ the power of the ultrasound generator, $P_{\text {gen }}$;

- $\quad$ the geometry of the system;

- $\quad$ the characteristics of the surfaces;

- the material of the surface and the possible formation of chemical substances.

As already discussed by the authors in a different paper [24], the modifications induced by the action of an acoustic field, and in particular by ultrasound action, can be explained in different ways for the three different kinds of fluid: gas, and liquid of two phases.

In the case of the gas, three phenomena of convective heat transfer alteration can be qualitatively identified: the dissipation into heat of the introduced vibrational energy (thermo-viscous dissipation of acoustic field); the alteration of the mean flow trajectories (acoustic streaming); the intensification of the conductive heat-transfer rate in the thermal boundary layer (acoustic modification of thermal boundary layer).

In the case of liquid, there are further conditions beyond the three previously mentioned conditions: the alteration and mixing of the fluid flow, in particular in the thermal boundary layer, through the implosion of low-pressure vapor bubbles (due to vaporous acoustic cavitation); a similar violent alteration of vaporous acoustic cavitation, but through the implosion of low-pressure gas bubbles (gaseous acoustic cavitation); the removal of fouling layers by the mechanical stress inducted by acoustic cavitation (anti-fouling effect of acoustic cavitation).

In the case of two phase fluid (for example pool boiling), all the phenomena exposed above are still present. Furthermore, a new peculiar phenomenon can be identified: the vapor bubble facilitated detachment on the heating (acoustic release of the vapor bubble).

As reported in the available literature and discussed by the authors in the aforementioned paper [24], the heat-transfer enhancement due to ultrasound can be largely different according to the various experimental analysis reported in the literature, varying from $10 \%$ up to $300 \%-400 \%$ in some particular favorable cases. In general, the most interesting enhancement can be obtained in the case of liquids in subcooling conditions, but the actual amount appears to be strongly dependent on the specific experimental situation.

The acoustic energy of an ultrasonic beam that passes through a liquid medium can be converted into different forms of energy: this conversion mechanism can be divided into a thermal part, related to o cavitation, and into a part that determines the motion of the liquid particles.

When the ultrasounds are absorbed by the medium, the energy is partially converted into heat: this leads to an increase of the temperature of the fluid. Moreover, an ultrasonic wave produces areas of compression and areas of rarefaction in the liquid; such changes in pressure determine the occurrence of cavitation. This causes oscillations and pulsations inside the liquid (this is referred to as stable cavitation), or the collapse of the gas bubbles themselves (this is called unstable cavitation), which leads to instantaneous and large increases in the temperature and in the pressure of the liquid.

Experimental studies have shown that unstable cavitation does not occur until the intensity of the acoustic field exceeds a limit value, which is also dependent on the particular experimental conditions. In the frequency range between 0.25 and $4 \mathrm{MHz}$, this limit has been set at $3 \mathrm{~kW} / \mathrm{m}^{2}$ and is directly proportional to the frequency (that is, the higher the frequency, the greater the intensity necessary to lead to unstable cavitation). 
Finally, when an ultrasound field propagates in a liquid, its particles take part in an oscillatory flow. If the particle oscillates in a direction parallel to that of the wall, the non-slip boundary conditions apply on the wall itself, since it is rigid, and the velocity of the liquid will be zero. Thus a steady-state fluid flow will be established in the boundary layer between the surface and the fluid particles at the interface, with a wide speed gradient. The most interesting phenomenon for our research, aiming at the investigation of ultrasounds from the point of view of their influence on heat transfer, is certainly stable cavitation.

The characteristic cavitation parameter is the cavitation number $\sigma$, defined as:

$$
\sigma=2 \frac{p_{0}-p_{v}}{\rho w^{2}}
$$

where $p_{0}$ is the ambient pressure, $p_{v}$ is the water vapor pressure, $\rho$ the fluid density and $w$ the velocity of the liquid. In order for cavitation to occur, a substrate is needed that acts as a nucleation center: this may be the surface of a container, impurities present in the liquid or other irregularities. The temperature has a considerable influence on cavitation, since it modifies the vapor pressure.

If the temperature increases, the higher vapor pressure promotes cavitation, at least until the saturation conditions are reached.

In general, cavitation is an undesired phenomenon and a source of problems. In devices such as pumps and propellers, cavitation causes a significant loss of efficiency, noise emission and component damage. The collapse of the cavitation bubbles in fact generates a large amount of noise and a very intense mechanical shock, which, if occurring often, can significantly reduce the life of propellers and impellers of pumps. Finally, cavitation causes friction and turbulence in the liquid, which leads to a further decrease in efficiency. In our case it is seen as a phenomenon that increases the efficiency of heat transfer.

\section{Experimental Studies on Heat-Transfer Enhancement: General Elements}

The critical issues encountered in the analysis of the equipment available in the field of applications are the following:

- Difficulty in modifying the acoustic parameters: the frequency is almost always fixed and often the power cannot be varied; geometry and fluid dynamics influence the actual power released by the generator. It would be useful to modify the generators in such a way that they always supply the set power;

- With the exception of some particular cases, the acoustic generation leads to high $P_{\mathrm{US}} / \Delta P_{\text {th }}$ ratios, where $P_{\mathrm{US}}$ is the total power given with the ultrasounds and $\Delta P_{\text {th }}$ represents the heat-transfer enhancement. This occurs because a large part of the acoustic energy is dissipated in the fluid before reaching the exchange surface and before altering it. The possibility could be assessed case by case to switch to mechanical generation or to bring the exchange surface as close as possible to the generation area.

Overall, it can be observed that in each of the considered cases it is rather difficult to identify the contribution of the single effects and, therefore, to draw general conclusions on the effect of the application of ultrasounds.

Concerning the direct enhancement of heat transfer, many limits clearly emerge, but the most important is that the use of ultrasonic power is not localized. In essence, the power is dissipated in the whole liquid and this also affects the liquid that is located near the exchange surface. Overall, it can be observed that in each of the considered cases it is rather difficult to be able to identify the contribution of the individual effects and, therefore, to draw general conclusions on the effect of the application of ultrasounds.

It is important to take into account the energy flows too. If the phenomenon to be obtained is dissipative, it risks being less efficient than that obtained by directly dissipating electricity in the liquid. 
Therefore, it is advisable to use mechanical generation or to bring the exchange surface as close as possible to the acoustic generation area.

Due to the marked non-linearity of the phenomena involved, it is advisable to operate with dimensions and geometries similar to those of the final applications. It would be also advisable to be able to set the acoustic parameters (frequency, power, etc.) independently of other operating conditions, geometries, etc.

For the aforementioned reasons, the authors have decided to develop a specific experimental apparatus in order to test the effect of ultrasounds on natural convection in a dielectric fluid. The experimental analysis is carried out to estimate the achievable increase of the convective heat-transfer coefficient between an electronic board and the fluid.

\section{Definition of the Experimental Setup and Measurement System}

In this section, the experimental setup is described with some details about the specific components and the measurement methodology.

\subsection{Experimental Setup}

The measurements are performed in an ultrasonic cleaning tank, with an internal volume of $12 \mathrm{~L}$. The tank used (Novatec model MU-12L), has a rectangular base and is made of stainless steel. Its dimensions are $300 \mathrm{~mm} \times 200 \mathrm{~mm} \times 230 \mathrm{~mm}$ and its capacity is 12 liters. The refrigerant fluid can be introduced into the tank and the same fluid is maintained at the imposed temperature by means of an external cooling system.

The tank is also used for ultrasound generation by means of 4 ultrasound transducers. The total power of the 4 ultrasound transducers, operating at $40 \mathrm{kHz}$ and placed underneath the bottom of the tank, is $500 \mathrm{~W}$.

The generation of ultrasonic waves is achieved by means of piezoelectric transducers (made of barium titanate or lead zirconate titanate), which transform high-frequency electrical energy into mechanical energy in the form of ultrasonic waves. The tank has a push-button panel (Figure 1) that allows the fine adjustment of some parameters, among which the ultrasound power, variable between 300 and $500 \mathrm{~W}$, their frequency, in steps of $25 \mathrm{~Hz}$ around the center frequency of $40 \mathrm{kHz}$, in order to optimize the resonance with the walls of the tank itself, and the duration of the ultrasound emission. The temperature of the fluid in the tank is measured with a thermocouple connected to the digital readout system.
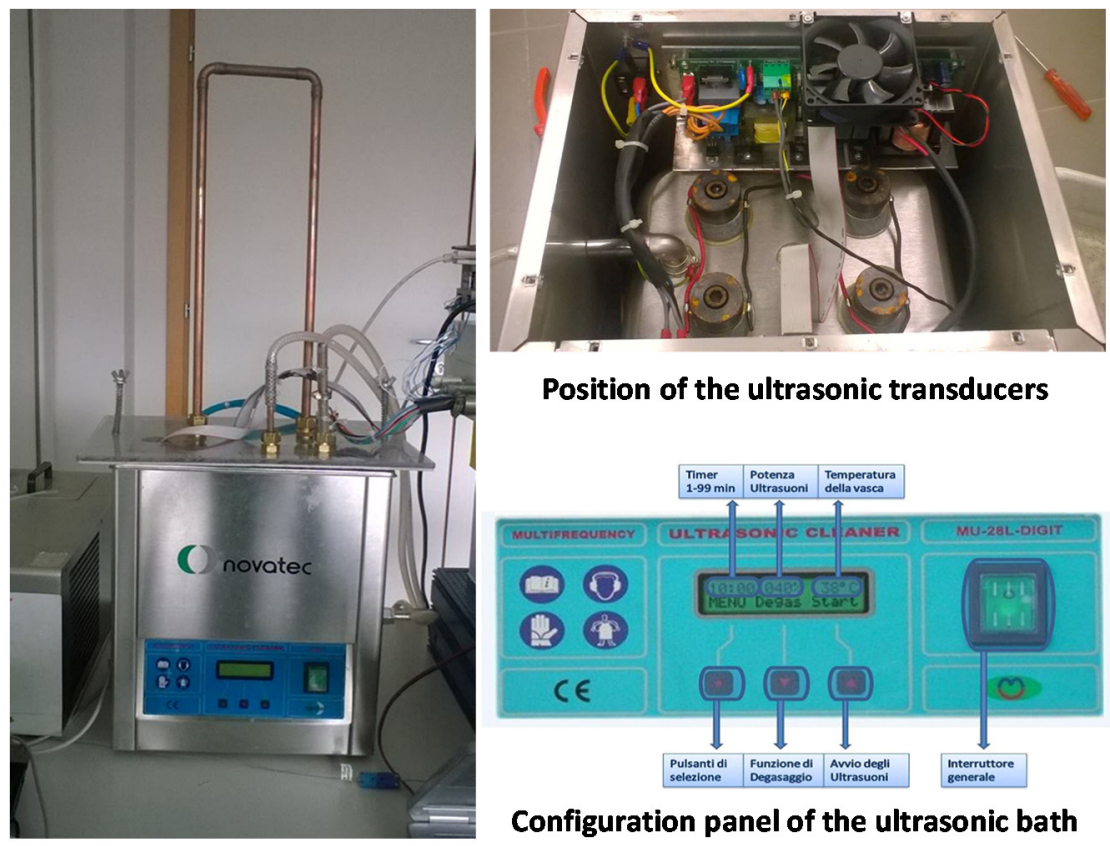

Position of the ultrasonic transducers

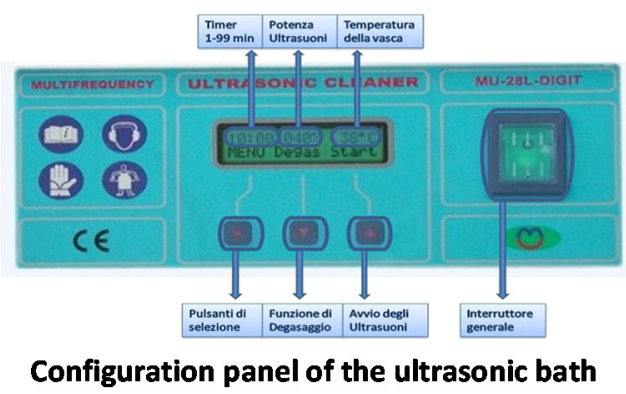

Figure 1. Ultrasound tank used in the experiments. 
The electronic test board to be introduced in the coolant liquid includes 9 TIP31C bipolar power transistors, operating at constant power. Each transistor is in good thermal contact with a PT100 temperature sensor. A control board stabilizes the temperature of the nine TIP31C. Figure 2 shows the position of the nine transistors in the test board and the control board. The control board provides the necessary power and performs part of the measurements.

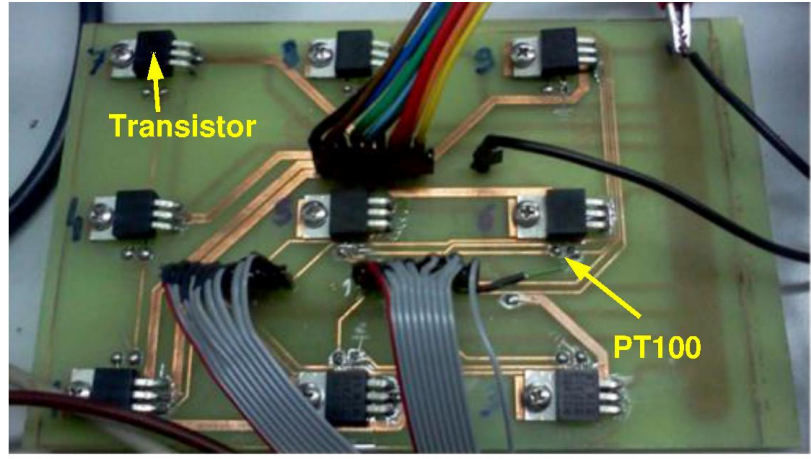

a)

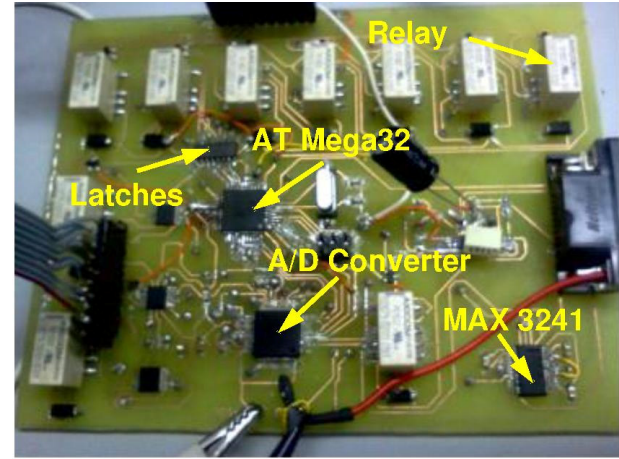

b)

Figure 2. The nine TIP31C transistors used as heaters (a) and control board (b).

\subsection{Measurement Strategy and Control System}

In order to keep the temperature of the power transistors constant, a digital control circuit has been implemented. It acquires the transistor temperature (measured with a PT100 platinum resistor in direct contact with the metallic part of each transistor) and then applies a base current determined by a PID (proportional, integral, derivative) algorithm (in which only the proportional term is actually significant).

The temperature of the test devices, is kept constant by automatically adjusting the dissipated power in each of them. Heat is transferred from the transistors to the dielectric fluid and from the dielectric fluid to cold water by means of a copper coil heat exchanger. When a steady-state condition is achieved, the temperature of the transistors $T_{\mathrm{T}}$ and that of the fluid $T_{\mathrm{f}}$ remain constant. The printed circuit board with the power transistors is held horizontal with the transistors facing down. The acoustic field in the dielectric fluid has been measured with a Bruel and Kjaer meter, obtaining value around 0.5 bar.

In order to keep the temperature of the power transistors at a constant value of $60^{\circ} \mathrm{C}$, a digital control circuit has been implemented. The control circuit uses the transistor temperature (measured with a PT100 platinum resistor in direct contact with the metallic part of each transistor) and then applies a base current determined with a PID algorithm, in which only the proportional term is significant.

During the experiments, the temperature of the transistors is held constant by means of the control algorithm, thus it is straightforward, for a given value of the temperature of the dielectric fluid, to compute the dissipated power (and hence the heat flux), from the collector current and the voltage drop between the collector and the emitter. The digital control algorithm has been implemented with an Atmel ATMega32 microcontroller, which cyclically scans the PT100 sensors, inserting each of them in the arm of a Wheatstone bridge, whose unbalance is measured via a precision differential amplifier base on a Burr-Brown INA321 instrumentation amplifier. The amplified output voltage of the Wheatstone bridge is digitized by the analog-to-digital converter (ADC) of the microcontroller.

The temperature is the obtained from the equation of the Wheatstone bridge and from the functional dependence of the PT100 resistance, $R$ on the temperature $T$ according to:

$$
R=R_{0}+a T+b T^{2}
$$


where

$$
\begin{gathered}
R_{0}=100 \Omega \\
a=3.908 \times 10^{-3}{ }^{\circ} \mathrm{C}^{-1} \\
b=-5.8019 \times 10^{-7}{ }^{\circ} \mathrm{C}^{-2}
\end{gathered}
$$

In the PID algorithm the integral and derivative actions are not active, since it was found that reliable operation within $\pm 0.3^{\circ} \mathrm{C}$ was obtained just with the proportional action. The currents in the TIP31C power transistors are determined by means of the voltages applied, via bias resistors, through the MCP6444 buffer, which is in turn fed by a MAX5621 16-bit digital-to-analog converter.

The particular structure of the measurement systems provides a motivation for choosing a particular refrigerant fluid such as FC 72. Moreover, the particular fluid has been selected due to the fact that the saturation temperature at atmospheric pressure $\left(56^{\circ} \mathrm{C}\right)$ is quite close to the maximum admissible temperature of the electronic components used for the experiments $\left(60^{\circ} \mathrm{C}\right)$. This allows various subcooling conditions. Indeed it has particularly favorable characteristics with respect to the operation of the electronic equipment: a relative permittivity $\varepsilon_{\mathrm{r}}=1.75$, which thus does not raise stray capacitance values significantly with respect to operation in air, a thermal conductivity of $0.057 \mathrm{~W} /(\mathrm{m} \mathrm{K})$, a critical temperature of $176^{\circ} \mathrm{C}$, a critical pressure of $1.83 \times 10^{6} \mathrm{~Pa}$, an electrical resistivity of $10^{13} \Omega \mathrm{m}$, and a breakdown field of $15 \mathrm{MV} / \mathrm{m}$. It would not be possible to use water for example, because of its conductivity (even deionized water would lose its insulating properties in the presence of any contaminant) and its very large relative permittivity.

Figure 3 provides a block diagram of the experimental apparatus summarizing the interaction among all the different components. The main power supply provides the collector voltage to the transistors through $0.1 \Omega$ shunt resistors. The "resistance block" is connected to the multimeter and it is used for the measurement of the temperature of the transistors. The voltage drop across the shunt resistors is measured by a multi-channel multimeter connected to a computer. The ultrasound source can be turned on and off independent of the operation of the transistor board. The temperature of the bath is kept constant with an external cooling system.

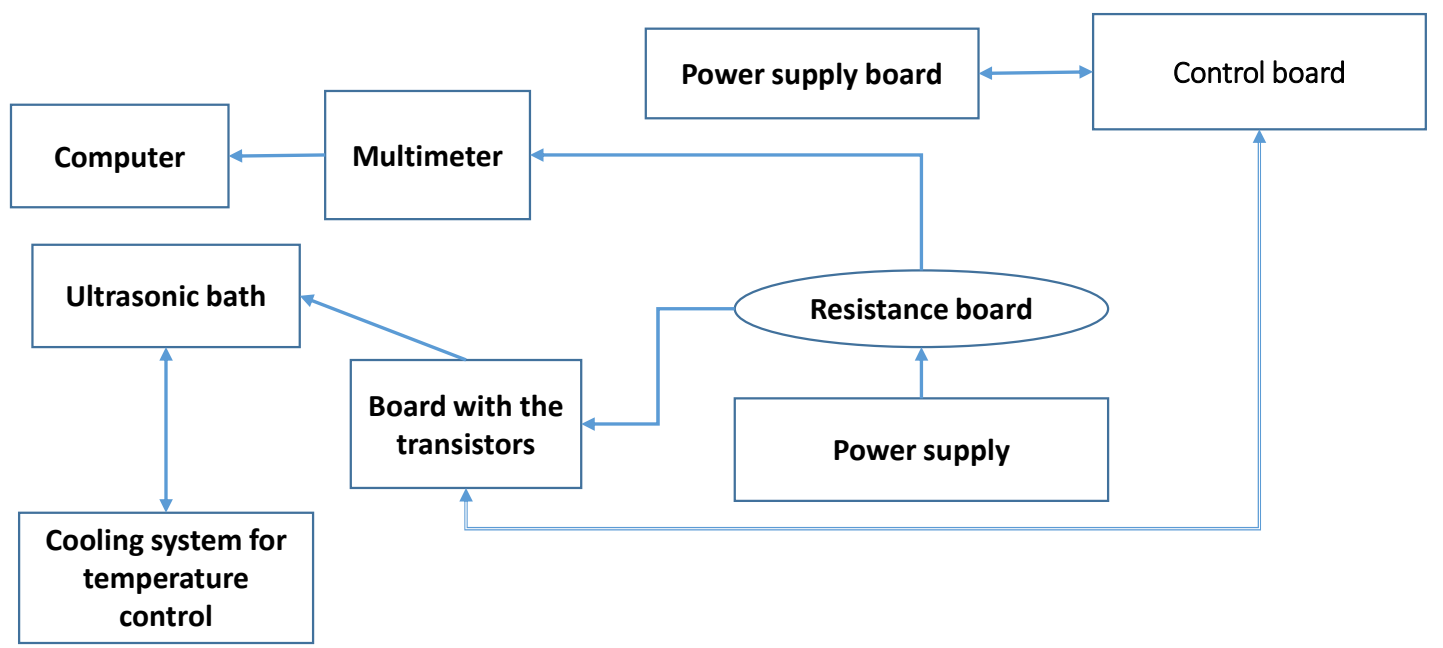

Figure 3. Block diagram of the experimental apparatus with the interaction among all the different components.

\section{Measurements for the Electronic Equipment Validation and Heat-Transfer Analysis}

In this section a detailed analysis of the experimental tests is presented: the first part covers the evaluation of the time constant of the experimental setup or, more properly, the time needed to reach a steady-state temperature for the power transistor in the test board, while the second part focuses on the the experimental data on the effect of ultrasounds on heat transfer. 


\subsection{Preliminary Operation}

Preliminary tests were performed by supplying all nine transistors with a drain voltage $6 \mathrm{~V}$.

In order to reduce noise in the acquisition of the PT100 resistance values, we chose to perform an average of every reading over 1000 acquisitions. The duration of the complete cycle over 9 transistors lasts about $1.8 \mathrm{~s}$. The temperature data for each transistor are sent on the serial port and they can be collected and inserted into a spreadsheet. We report the temperature data for a transistor during the switch-on transient (Figure 4): after an overshoot, the temperature reaches the set point value (in this case of $40{ }^{\circ} \mathrm{C}$ ) in a few tens of seconds, as clearly evidenced in Figure $4 \mathrm{a}$. In Figure $4 \mathrm{~b}$ we report instead the temperature stability during the steady-state condition, which is well within $\pm 0.1^{\circ} \mathrm{C}$.
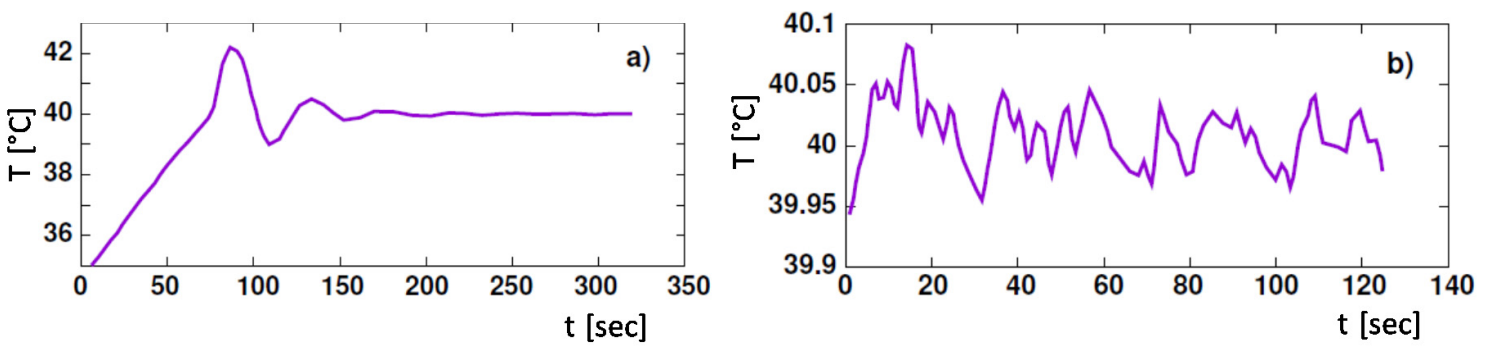

Figure 4. Plot of the temperature transient with respect to time at switch-on (a) and temperature vs. time in "steady state conduction" (b).

\subsection{Thermal Measurements and Data Analysis}

The experimental analysis has been carried out operating in a steady-state condition, with the temperatures of the transistors held constant by the control circuit and that of the refrigerant fluid held constant by tuning the FC72 flux in the cooling coil.

The thermal power dissipated by each transistor can be measured from the product of the applied collector-emitter voltage (adjustable in the interval between 5 and $18 \mathrm{~V}$ ) and the collector current, which is evaluated from the voltage drop across a $0.15 \Omega$ resistor in series with the power supply. In order to obtain an estimate of the variation of the convective heat transfer coefficient as a result of the application of ultrasounds, we measure the power that the transistors need to dissipate to maintain a constant temperature with and without ultrasound waves. In order to perform the measurement in a condition without global cavitation, it is undertaken before each test ultrasonic waves are intermittently applied (with a built-in function of the tank), thereby removing gases present in the dielectric fluid. The printed circuit board with the TIP31C transistors is placed in the tank with the component side facing down, in such a way that it has direct exposure to the action of the ultrasonic transducers. The collector-emitter voltage was set at $15 \mathrm{~V}$ in the experiments that we report in the following.

In the actual measurements, the transistor temperature was always kept at a constant value of $60{ }^{\circ} \mathrm{C}$ (instead of the $40{ }^{\circ} \mathrm{C}$ considered for the preliminary tests), while the temperature of the fluid was maintained at a value between 28 and $44^{\circ} \mathrm{C}$, obtaining a subcooling effect, $\Delta T_{\text {sub variable between } 12}$ and $28 \mathrm{~K}$, being the saturation temperature of FC72 at atmospheric pressure equal to $56{ }^{\circ} \mathrm{C}$.

In order to obtain similar operating conditions (i.e., comparable values of the heat transfer coefficient), the power given to the transistors has been opportunely reduced in proportion with the subcooling degree, $\Delta T_{\text {sub }}$ meaning the temperature difference between the saturation temperature and the operating temperature of the fluid.

An error analysis was undertaken before and after the tests: even if the error on the single values can be easily controlled and described before, the accuracy of the data indirectly obtained of the heat transfer coefficient, obtained combining power and temperature difference, is difficult to estimate, mainly due to the reduced values of temperature difference. Anyway, it can be considered in the range between $5 \%$ and $30 \%$, depending on the subcooling. Obviously the values obtained with quite high subcooling degrees are more accurate. The values obtained with a subcooling degree of $12{ }^{\circ} \mathrm{C}$ are surely less accurate than those obtained at $28^{\circ} \mathrm{C}$. 
For a quantitative measurement of the effect of the action of ultrasounds on the heat-transfer coefficient, an enhancement factor EF is used. This is defined as the ratio between the heat-transfer coefficient estimated in the case of the presence of ultrasounds, $h_{U S}$ and the value obtained without ultrasounds, $h$, for the same hydrodynamic configuration. In an equivalent way, it is possible to define the ratio of the enhanced value of the heat-transfer coefficient and the basic value obtained without ultrasounds. The two enhancement factors are defined, respectively as:

$$
\begin{gathered}
E F=\frac{h_{U S}}{h} \\
\frac{\Delta h}{h}=\frac{h_{U S}-h}{h}
\end{gathered}
$$

The positive effect of ultrasounds can be appreciated, comparing the results reported in Figures 5 and 6 , in which the results of five different experiments, obtained with different values of the temperature of each transistors and of the resulting heat flux. In particular, we plot the estimated value of the heat-transfer coefficient in the reference case (Figure 5) and in the presence of ultrasound (Figure 6). In this second case it is possible for the transistors dissipate a higher power for the same temperature difference between the transistors and the coolant fluid.

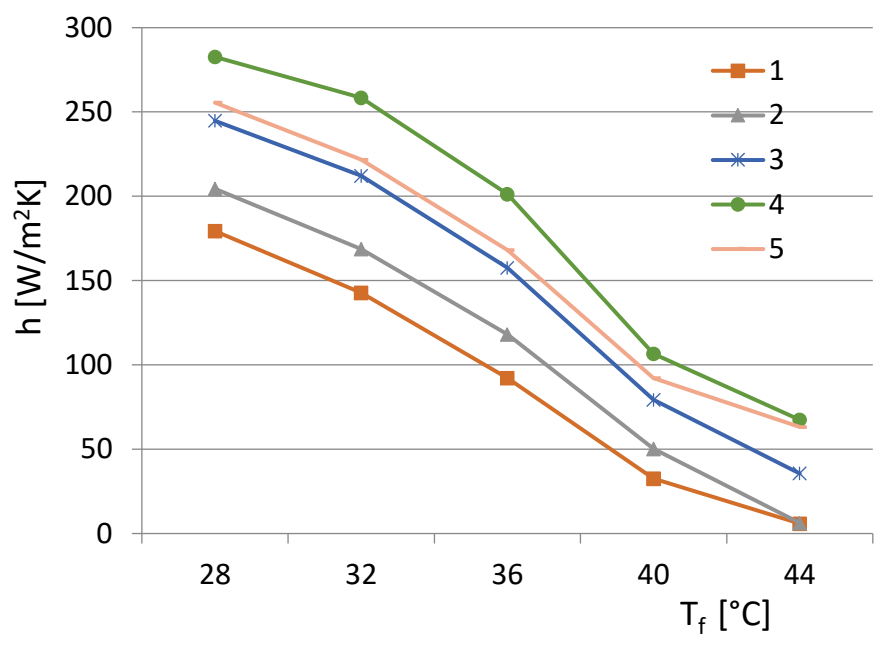

Figure 5. Heat flux removed as a function of the temperature without ultrasound action (results of five different experiments).

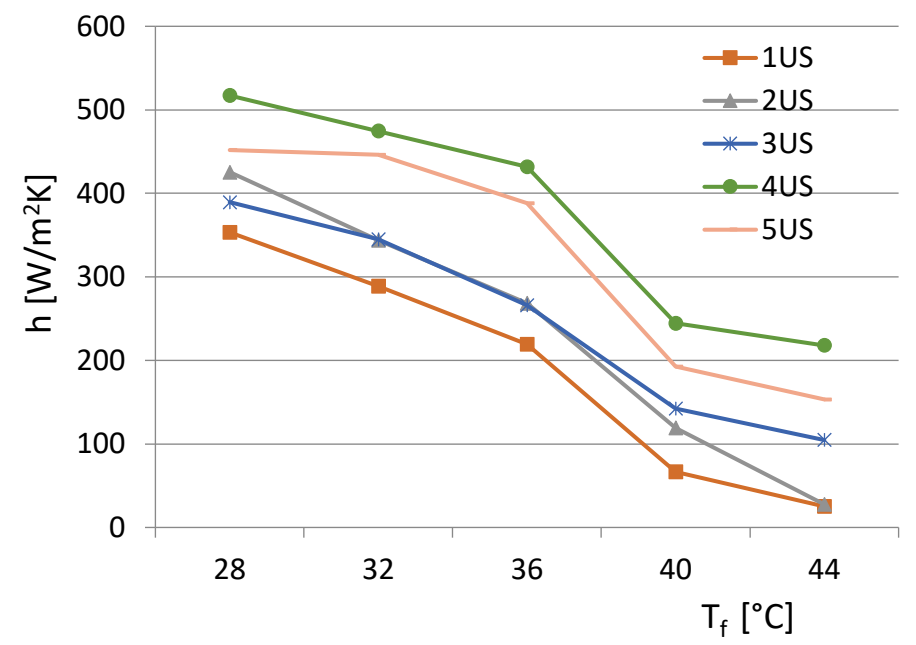

Figure 6. Heat flux removed as a function of the temperature of the fluid with ultrasound action (result of five different experiments corresponding of those of Figure 5). 
Both figures show the power dissipated with and without ultrasounds in two sets of experiments. The data can be considered meaningful although some fluctuation can be observed due to the small values of the temperature differences between the transistors and the subcooled refrigerant. We present average values over the nine transistors, due to the existence of some fluctuations among the different transistors on the board, probably resulting from the non-uniform ultrasound pressure field.

As can be observed, the values are different, but a general improvement determined by ultrasound action can be clearly noticed. The heat-transfer coefficient in particular can be estimated with a specific application of Newton's Law:

$$
\begin{gathered}
q=V I=h A\left(T_{T}-T_{f}\right) \\
h=\frac{q}{\Delta T}
\end{gathered}
$$

where $V$ is the voltage and $I$ the current supplied to each transistor, $A$ is the heat transfer area of each transistor, $T_{T}$ is an average temperature measured on the nine transistors and $T_{f}$ the temperature of the fluid.

An average value of the heat-transfer coefficient obtained in the experimental analysis between the normal case and the case in which an ultrasound action is present is summarized in Table 1. A simple analysis of the data contained in Table 1 provides evidence that the increase of the heat-transfer coefficient obtained by means of the ultrasound action can be in some cases quite remarkable, although not exactly constant.

Table 1. Average dissipated power in each transistor and computed relative variation of the heat-transfer coefficient for different subcooling values.

\begin{tabular}{cccc}
\hline$\Delta \boldsymbol{T}_{\text {sub }}[\mathrm{K}]$ & $\boldsymbol{P}_{\mathbf{t}}[\mathrm{W}]$ & $\boldsymbol{E F}$ & $\boldsymbol{\Delta} \boldsymbol{h} / \boldsymbol{h}$ \\
\hline 12 & 1.89 & 3.476 & 2.476 \\
16 & 2.43 & 2.223 & 1.123 \\
20 & 2.52 & 2.260 & 1.160 \\
24 & 3.00 & 1.907 & 0.907 \\
28 & 3.97 & 1.848 & 0.848 \\
\hline
\end{tabular}

The power dissipated by a single transistor (averaged over all the transistors) is reported in Table 1 in the reference case (without ultrasound action) for each experimental condition, characterized by the value of the subcooling temperature $\left(\Delta T_{\mathrm{sub}}\right)$. Obviously, the action of the ultrasounds allows increasing the operating power of the devices (i.e. the thermal power removed by the bath) at a given temperature difference between the devices and the coolant. In the third and in the fourth columns of Table 1 the average value of the enhancement factor and of the relative increase of the heat-transfer coefficient, obtained considering the results of the experiments reported in Figures 5 and 6, are reported, according to the definitions given in Equations (3) and (4).

A general trend toward the decrease of the heat-transfer enhancement with the increase of the subcooling level, although with some oscillations (probably due to measurement uncertainties), is observed. This is clear because the effect of ultrasounds is higher in conditions close to those of pool boiling, at a temperature of $44{ }^{\circ} \mathrm{C}$ of the refrigerant. In this case, the mechanism of acoustic cavitation is clearly evident as well as the beneficial effect of ultrasounds on the heat transfer. On the other hand, the increase of the heat-transfer coefficient appears to be sensibly lower (but in any case is always relevant, because it is about $85 \%$ ) at a subcooling value of $28{ }^{\circ} \mathrm{C}$, thus with the refrigerant at $28^{\circ} \mathrm{C}$. In this case, the mechanism of acoustic streaming is the only one that is relevant, while the effect of acoustic cavitation is minor. Figure 7 summarizes the results of Table 1. 


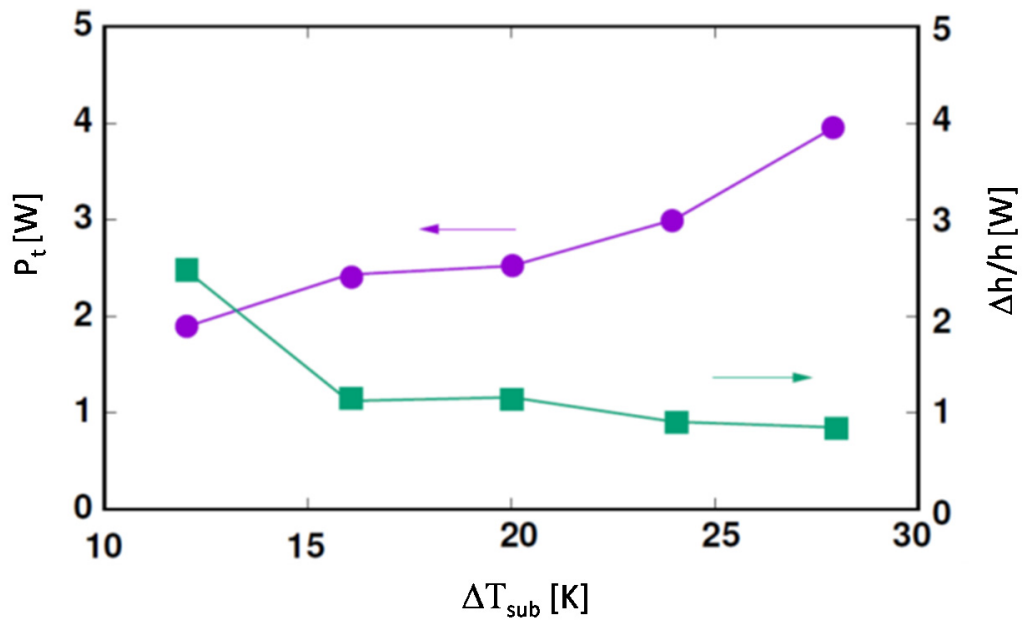

Figure 7. Plot based on the parameters shown in Table 1.

At the end of the tests, the condition of the transistor board was checked. It was found to be free from any damage, thus confirming that the investigated cooling technique is absolutely suitable for the thermal control of electronic equipment.

\section{Conclusions}

We have presented an experimental investigation on the effect of ultrasound application to increase the heat-transfer coefficient in the case of natural convection in a dielectric fluid. After a preliminary analysis of the effect of ultrasound on heat-transfer enhancement, we have described the results of an experimental analysis aiming at the evaluation of the enhancements that can be obtained for the convective heat-transfer coefficient between an electronic component and the FC72 dielectric fluid with the application of ultrasound at a fixed frequency of $40 \mathrm{kHz}$.

The analysis of the experimental results obtained shows a positive effect of ultrasound in all the analyzed conditions. An increase of the heat-transfer coefficient between $80 \%$ and $250 \%$ can be obtained in the different conditions. As expected from the available literature, the heat-transfer enhancement appears to be relevant in the case of reduced value of the subcooling degree, while an overall degradation in the heat removal performance has been observed as a function of the amount of subcooling and this confirms the fact that the main contribution to the heat-transfer enhancement is given by the acoustic cavitation.

In the near future we plan to perform more detailed measurements, in particular with a better resolution in terms of the amount of subcooling, also implementing a fully automated control of the fluid temperature. The authors also plan to study how the heat transfer coefficient depends on the angle formed by the electronic board with the direction of propagation of the ultrasound waves.

Author Contributions: Conceptualization, A.F. and C.B.; methodology, A.F. and C.B.; experimental setup, C.B. and M.M.; validation, C.B., M.M.; investigation, C.B.; data curation, A.F. and C.B.; writing-original draft preparation, A.F.; writing-review and editing, A.F., C.B., M.M.; supervision, A.F. All authors have read and agreed to the published version of the manuscript.

Funding: This research received partial support by the Italian Ministry of Education and Research (MIUR) in the framework of the Crosslab project (Department of Excellence).

Acknowledgments: M.M. acknowledge partial support by the Italian Ministry of Education and Research (MIUR) in the framework of the Crosslab project (Department of Excellence).

Conflicts of Interest: The authors declare no conflict of interest. 


\section{References}

1. Martinelli, R.C.; Boelter, L.M.K. The Effect of Vibration upon the Free Convection from a Horizontal Tube. In Proceedings of the 5th International Congress for Applied Mechanics, Cambridge, MA, USA, 12-16 September 1938; Volume 578.

2. Fand, R.M.; Roos, J.; Cheng, P.; Kaye, J. The local heat-transfer coefficient around a heated horizontal cylinder in an intense sound field. J. Heat Transf. 1962, 84, 245-250. [CrossRef]

3. Kubanskii, P.N. Currents around a heated solid in a standing acoustic wave. Trans. USSR Acad. Sci. 1952, 82,585 .

4. Holman, J.P.; Mott-Smith, T.P. The effect of high constant pressure sound fields on free convection heat transfer from a horizontal cylinder. J. Aerosp. Sci. 1959, 20, 188. [CrossRef]

5. Anantanarayanan, R.; Ramachandran, A. Effect of vibration on heat transfer from a wire to air in parallel flow. ASME J. Heat Transf. 1958, 80, 1426-1432.

6. Jackson, T.W.; Harrison, W.B.; Boteler, W.C. Free convection, forced convection, and acoustic vibrations in a constant temperature vertical tube. ASME J. Heat Transf. 1959, 81, 68-74. [CrossRef]

7. Lemlich, R.; Hwu, C.K. The effect of acoustic vibration on forced convective heat transfer. AIChE J. 1961, 71, 102-106. [CrossRef]

8. Fand, R.M.; Cheng, P. The influence of sound on heat transfer from a cylinder in crossflow. Int. J. Heat Mass Transf. 1963, 6, 571-596. [CrossRef]

9. Larson, M.B.; London, A.L. A study of the effect of ultimate vibrations on convective heat transfer to liquids. ASME 1962, 62, 62-68.

10. Deaver, F.K.; Penney, W.R.; Jefferson, T.B. Heat transfer from an oscillating horizontal wire to water. J. Heat Transf. 1962, 84, 251-254. [CrossRef]

11. Fand, R.M. The influence of acoustic vibrations on heat transfer by natural convection from a horizontal cylinder to water. J. Heat Transf. 1965, 87, 309-310. [CrossRef]

12. West, F.B.; Taylor, A.T. The effect of pulsations on heat transfer-turbulent flow of water inside tubes. Chem. Eng. Prog. 1952, 48, 39-43.

13. Scanlan, J.A. Effects of normal surface vibration on laminar forced convective heat transfer. Ind. Eng. Chem. 1958, 50, 1565-1568. [CrossRef]

14. Raben, I. The use of acoustic vibrations to improve heat transfer. In Proceedings of the 1961 Heat Transfer and Fluid Mechanica Institute, Los Angeles, CA, USA, 19-21 June 1961; pp. 90-97.

15. Bergles, A.E. The influence of flow vibrations on forced-convection heat transfer. J. Heat Transf. 1964, 86, 559-560. [CrossRef]

16. Isakoff, S.E. Effect of an Ultrasonic Field on Boiling Heat Transfer-Exploratory Investigation; Heat Transfer and Fluid Mechanics Institute, Stanford University Press: Palo Alto, CA, USA, 1956; pp. 1-15.

17. Nangia, K.K.; Chon, W.Y. Some observations on the effect of interfacial vibration on saturated boiling heat transfer. AIChE J. 1967, 13, 872-876. [CrossRef]

18. Park, K.A.; Bergles, A.E. Ultrasonic enhancement of saturated and subcooled pool boiling. Int. J. Heat Mass Transf. 1988, 31, 664-667. [CrossRef]

19. Baffigi, F.; Bartoli, C. Effects of ultrasounds on the heat transfer enhancement from a circular cylinder to distilled water in subcooled boiling. J. Therm. Sci. Eng. Appl. 2011, 3, 011001. [CrossRef]

20. Bartoli, C.; Baffigi, F. Use of ultrasonic waves in subcooling boiling. Appl. Therm. Eng. 2012, 47, 95-110. [CrossRef]

21. Duan, X.L.; Wang, X.Y.; Wang, G.; Chen, Y.Z.; Qiu, X.Q. Experimental study on the influence of ultrasonic vibration on heat transfer and pressure drop in heat exchanger tubes. Petro-Chem. Equip. 2004, 33, 1-4.

22. Bulliard-Sauret, O.; Ferrouillat, S.; Vignal, L.; Memponteil, A.; Gondrexon, N. Heat transfer enhancement using $2 \mathrm{MHz}$ ultrasound, Ultrason. Sonochem 2017, 39, 262-271. [CrossRef]

23. Franco, A.; Bartoli, C. The ultrasounds as a mean for the enhancement of heat exchanger performances: An analysis of the available data. J. Phys. Conf. Ser. 2019, 1224, 012035. [CrossRef]

24. Franco, A.; Bartoli, C. Heat transfer enhancement due to acoustic fields: A methodological analysis. Acoustics 2019, 1, 281-294. [CrossRef] 
25. Baffigi, F.; Bartoli, C. Heat Transfer Enhancement from a Circular Cylinder to Distilled Water by Ultrasonic Waves: Preliminary Remarks. In Proceedings of the 7-th Word Conference on Experimental Heat Transfer (ExHFT-7), Krakow, Poland, 28 June-3 July 2009.

26. Baffigi, F.; Bartoli, C. Heat transfer enhancement from a circular cylinder to distilled water by ultrasonic waves in subcooled boiling conditions. In Proceedings of the Interdisciplinary Transport Phenomena VI: Fluid, Thermal; Biological, Materials and Space Sciences (ITP09), Volterra, Italy, 4-9 October 2009.

(C) 2020 by the authors. Licensee MDPI, Basel, Switzerland. This article is an open access article distributed under the terms and conditions of the Creative Commons Attribution (CC BY) license (http://creativecommons.org/licenses/by/4.0/). 